\title{
Rare semileptonic charm D decays and possible new physics in charm
}

\section{Svjetlana Fajfer ${ }^{a, b, *}$}

${ }^{a}$ Faculty of Mathematics and Physics, University of Ljubljana, Jadranska 19, 1000 Ljubljana, SloveniaInstitution

${ }^{b}$ Jožef Stefan Institute,

Jamova 39, 1000 Ljubljana, Slovenia

E-mail: svjetlana.fajfer@ijs.si

I review Standard Model contributions to $c \rightarrow u l^{+} l^{-}$and $c \rightarrow u$ invisibles transitions in the exclusive rare $D \rightarrow \mathrm{Pl}^{+} l^{-}, D \rightarrow P_{1} P_{2} l^{+} l^{-}, D \rightarrow$ missing energy, and $D \rightarrow P$ missing energy decays. Then, I discuss New Physics effects in these processes, using low-energy existing constraints, as the experimental bound on $\mathcal{B}\left(D^{0} \rightarrow \mu^{+} \mu^{-}\right), D^{0}-\bar{D}^{0}$ oscillations. I compare them with the contributions allowed by the experimental limits on the rates for $D \rightarrow P l^{+} l^{-}, D \rightarrow P_{1} P_{2} l^{+} l^{-}$, and $D \rightarrow$ missing energy decays.

*** 10th International Workshop on Charm Physics (CHARM2020), ***

***31 May - 4 June, 2021 ***

*** Mexico City, Mexico - Online ***

${ }^{*}$ Speaker 


\section{Introduction}

The presence of physics beyond the Standard Model (SM) is more likely to be seen in the processes with down-like quarks. The appearance of New Physics (NP) in up-like quarks is much more challenging to detect. Although charm mesons are experimentally studied at each B-meson factory, at LHCb experiment, BESIII, flavour changing charm mesons decays driven by neutral current are difficult to approach theoretically and experimentally [1-9]. The search for NP in rare charm decay is biased by many non-charm resonances in the vicinity of D mesons masses. Also, the GIM mechanism plays a significant role in charm flavour changing neutral current (FCNC) dynamics. The interplay of CKM parameters and masses of down-like quarks leads to strong suppression of all FCNC in D meson processes. The main issue is how to isolate information on short-distance dynamics, either within SM or in its extensions. The separation of short from long-distance contributions is a longstanding issue in rare charm decays. A lot of discussion was devoted to these problems in the papers [6-9].

The B-meson anomalies indicate deviations from the SM prediction on the level $3-4 \sigma$ (for the latest review see [10]). From the study of NP one can set the scale of NP to be $3 \mathrm{TeV}$ for the $R_{D^{(*)}}$ anomaly and $\sim 30 \mathrm{TeV}$ for $R_{K^{(*)}}$ [11]. However, in the decay $D_{s} \rightarrow \ell v_{\ell} \mathrm{NP}$ at scale of 3 $\mathrm{TeV}$ has almost insignificant effect of $\sim 1 \%$ in the decay width. In Section 2 I introduce effective Lagrangians for $c \rightarrow u \ell \ell$. Section 3 is devoted to study of NP in $D^{+} \rightarrow \pi^{+} \mu^{+} \mu^{-}, D \rightarrow P_{1} P_{2} \mu^{+} \mu^{-}$, while Section 4 is dedicated to D-meson decays to invisible fermions. Section 5 contains a brief summary and outlook.

\section{Effective Lagrangians for $c \rightarrow u \ell^{+} \ell^{-}$and $c \rightarrow u v \bar{v}$ decays and NP}

The semileptonic $c \rightarrow u \ell^{+} \ell^{-}$and $c \rightarrow u v \bar{v}$ decay dynamics is described by the following effective Hamiltonian [5, 6, 8]

$$
\mathcal{L}_{\mathrm{eff}}=\frac{4 G_{F}}{\sqrt{2}} \frac{\alpha_{e}}{4 \pi}\left[\sum_{k=7,9,10}\left(C_{k} O_{k}+C_{k}^{\prime} O_{k}^{\prime}\right)+\sum_{i j}\left(C_{L}^{i j} Q_{L}^{i j}+C_{R}^{i j} Q_{R}^{i j}\right)\right],
$$

where the dimension six operators $O_{k}$ for di-lepton and $Q_{L / R}^{i j}$ for di-neutrino modes are given as

$$
\begin{array}{ccc}
O_{7} & =\frac{m_{c}}{e}\left(\bar{u}_{L} \sigma_{\mu \nu} c_{R}\right) F^{\mu \nu}, & O_{7}^{\prime}=\frac{m_{c}}{e}\left(\bar{u}_{R} \sigma_{\mu \nu} c_{L}\right) F^{\mu \nu}, \\
O_{9} & =\left(\bar{u}_{L} \gamma_{\mu} c_{L}\right)\left(\bar{l} \gamma^{\mu} \ell\right), & O_{9}^{\prime}=\left(\bar{u}_{R} \gamma_{\mu} c_{R}\right)\left(\bar{l} \gamma^{\mu} \ell\right), \\
O_{10} & =\left(\bar{u}_{L} \gamma_{\mu} c_{L}\right)\left(\bar{l} \gamma^{\mu} \gamma_{5} \ell\right), & O_{10}^{\prime}=\left(\bar{u}_{R} \gamma_{\mu} c_{R}\right)\left(\bar{l} \gamma^{\mu} \gamma_{5} \ell\right), \\
Q_{L}^{i j}=\left(\bar{u}_{L} \gamma_{\mu} c_{L}\right)\left(\bar{v}_{L j} \gamma^{\mu} v_{L i}\right), & Q_{R}^{i j}=\left(\bar{q}_{R} \gamma_{\mu} c_{R}\right)\left(\bar{v}_{L j} \gamma^{\mu} v_{L i}\right) .
\end{array}
$$

$F^{\mu \nu}$ is the electromagnetic field strength tensor and $\sigma^{\mu \nu}=\frac{1}{2}\left[\gamma^{\mu}, \gamma^{\nu}\right]$. In the primed operators $O_{i}^{\prime}$ right/left-handed $R / L$ chiral quark fields, are simply interchanged from $O_{i}$. SM contributions to the effective coefficients of $O_{7}$ and $O_{9}$ are generated by the charged-current four-quark operators as is explained in detail in [6-9]. The SM contributions, including two-loop virtual corrections to the SM contributions depend on the di-lepton invariant mass squared, $q^{2}$, as given in $[5,12]$. As discussed in [8,9] the non-vanishing SM contributions relevant to $c \rightarrow u \ell^{+} \ell^{-}$are of the order of few permille for $\left|C_{7}^{\text {eff }}\right|$ and few percent for $\left|C_{9}^{\text {eff }}\right|$ above $q^{2}>0.1 \mathrm{GeV}^{2}$. Note that the GIM-mechanism 


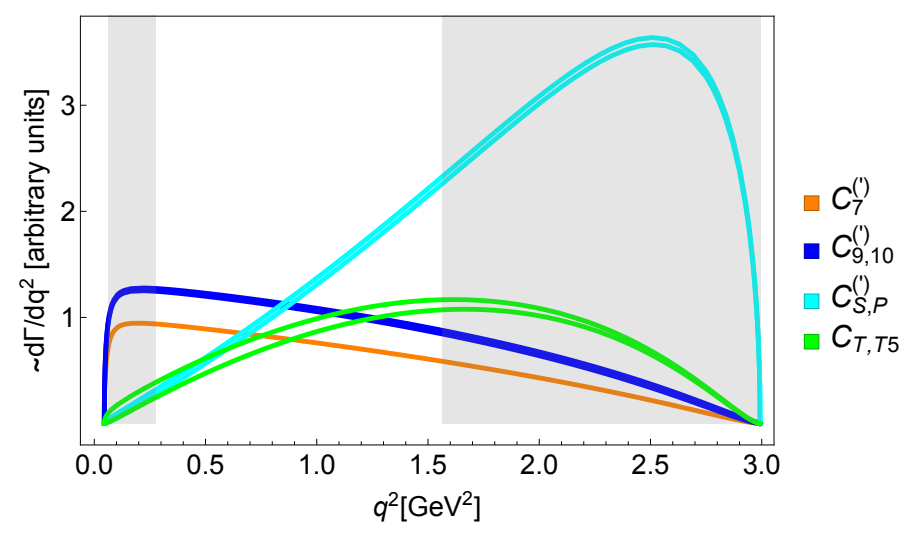

Figure 1: Comparison of short-distance spectrum sensitivities to different Wilson coefficients. Grey regions indicate the LHCb experimental low- and high $-q^{2}$ bins.

implies $C_{10}^{\mathrm{SM}}=0$. Contributions of primed operators are significantly suppressed, allowing us to neglect them.

\section{Search for NP in $D^{+} \rightarrow \pi^{+} \mu^{+} \mu^{-}, D \rightarrow P_{1} P_{2} \mu^{+} \mu^{-}$}

Experimentally the upper bound on the branching ratio $\mathcal{B}\left(D^{0} \rightarrow \mu^{+} \mu^{-}\right)<6.2(7.6) \times 10^{-9}$ was first presented by $\mathrm{LHCb}$ [13]. LHCb collaboration established limits on the branching fractions in several di-lepton invariant mass bins in $\left(D^{+} \rightarrow \pi^{+} \mu^{+} \mu^{-}\right)<7.3(8.3) \times 10^{-8}$ [14]. Extending the effective Lagrangian in (2) by the scalar, pseudoscalar and tensor operators $O_{S}=\frac{e^{2}}{(4 \pi)^{2}}\left(\bar{u} P_{R} c\right)(\bar{\ell} \ell)$, $O_{P}=\frac{e^{2}}{(4 \pi)^{2}}\left(\bar{u} P_{R} c\right)\left(\bar{\ell} \gamma_{5} \ell\right), O_{T}=\frac{e^{2}}{(4 \pi)^{2}}\left(\bar{u} \sigma_{\mu \nu} c\right)\left(\bar{\ell} \sigma^{\mu \nu} \ell\right)$, and $O_{T 5}=\frac{e^{2}}{(4 \pi)^{2}}\left(\bar{u} \sigma_{\mu \nu} c\right)\left(\bar{\ell} \sigma^{\mu \nu} \gamma_{5} \ell\right)$ the bounds on the appropriate Wilson coefficients are presented in Fig. 1 from the calculation in [5]. However, it was discussed already in [5] that better bounds can be derived from the experimental bound on $D^{0} \rightarrow \mu^{+} \mu^{-}$, approximately by factor 10 .

The various scenarios of NP were discussed in the literature in particular as solutions to the B-meson anomalies. Following work of $[5,8]$ the bounds on leptoquark contributions, Two-Higgs doublet model type III, $Z^{\prime}$-model are derived and presented in Table 2.

LHCb collaboration in [15] found

$$
\begin{aligned}
\left.\mathcal{B}\left(D^{0} \rightarrow \pi^{+} \pi^{-} \mu^{+} \mu^{-}\right)\right|_{[0.565-0.950] \mathrm{GeV}} & =(40.6 \pm 5.7) \times 10^{-8}, \\
\left.\mathcal{B}\left(D^{0} \rightarrow \pi^{+} \pi^{-} \mu^{+} \mu^{-}\right)\right|_{[0.950-1.100] \mathrm{GeV}} & =(45.4 \pm 5.9) \times 10^{-8}, \\
\left.\mathcal{B}\left(D^{0} \rightarrow K^{+} K^{-} \mu^{+} \mu^{-}\right)\right|_{[>0.565] \mathrm{GeV}} & =(12.0 \pm 2.7) \times 10^{-8},
\end{aligned}
$$

On the theory side, analyses of the paper [9] indicate that the SM angular distribution in semileptonic four-body $D$-decays is significantly simpler than in $B$-decays. Namely, the longdistance dominance in charm simplifies the picture. The authors of [9] discovered a few angular coefficients that can serve as null tests of the SM. In the same study, NP-induced CP violation were studied and suggested to be searched by experiment. However, the LHCb found that the CP asymmetries, the forward-backward asymmetry of the di-muon pair, the triple-product asymmetry, and the charge-parity-conjugation asymmetry are consistent with SM predictions [16]. 


\begin{tabular}{|c|c|c|}
\hline Model & Effect & Size of the effect \\
\hline $\begin{array}{l}\text { Scalar leptoquark } \\
(3,2,7 / 6)\end{array}$ & $\begin{array}{l}C_{S}, C_{P}, C_{1}^{\prime}, C_{p}^{\prime}, C_{T}, C_{T 5}, \\
C_{9}, C_{10}, C_{9}^{\prime}, C_{10}^{\prime}\end{array}$ & $\mathrm{V}_{\mathrm{cb}} \mathrm{V}_{\mathrm{ub}}\left|\mathrm{C}_{9}, \mathrm{C}_{10}\right|<0.34$ \\
\hline $\begin{array}{l}\text { Vector leptoquark } \\
(3,1,5 / 3)\end{array}$ & $\mathrm{C}_{9}^{\prime}=\mathrm{C}_{10^{\prime}}$ & $\mathrm{V}_{\mathrm{cb}} \mathrm{V}_{\mathrm{ub}}\left|\mathrm{C}_{9^{\prime},}, \mathrm{C}_{10^{\prime}}\right|<0.24$ \\
\hline $\begin{array}{l}\text { Two Higgs doublet } \\
\text { model type III }\end{array}$ & $C_{S}, C_{p}, C_{S}^{\prime}, C_{p}^{\prime}$ & $\begin{array}{l}V_{c b} V_{u b}\left|C_{s}-C_{s^{\prime}}^{\prime}\right|<0.005 \\
V_{c b} V_{u b}\left|C_{p}-C_{p}^{\prime}\right|<0.005\end{array}$ \\
\hline$Z^{\prime}$ model & $C_{9}^{\prime}, C_{10}^{\prime}$ & $\begin{array}{l}\mathrm{V}_{\mathrm{cb}} \mathrm{V}_{\mathrm{ub}}\left|\mathrm{C}_{9^{\prime}},\right|<0.001 \\
\mathrm{~V}_{\mathrm{cb}} \mathrm{V}_{\mathrm{ub}}\left|\mathrm{C}_{10^{\prime}}\right|<0.014\end{array}$ \\
\hline
\end{tabular}

Figure 2: Wilson coefficients calculated for different models of NP, following [5].

\section{Charm meson decays to invisible fermions}

Recently, a new detailed study of $c \rightarrow u v \bar{v}$ appeared in Ref. [7]. As well as in the case with the charged di-lepton pair in the final state, the amplitude for $c \rightarrow u v \bar{v}$ is a subject of severe Glashow-Iliopoulos-Maiani suppression. The authors of [17] found out that in the SM branching ratio $\mathcal{B}\left(D^{0} \rightarrow v \bar{v}\right)=1.1 \times 10^{-31}$. They also suggested that in searches for a dark matter candidate, it might be important to investigate the process with $\chi \bar{\chi} \gamma$ in the final state since a massless photon eliminates the helicity suppression. In our study [18] we also determine branching ratios for such decay modes. The authors of Ref. [7] computed the expected event rate for the charm hadron decays to a final hadronic state and neutrino - anti-neutrino states. They suggested that in experiments like Belle II, these processes can be seen. Also future FCC-ee might be able to detect branching ratios of $O\left(10^{-6}\right)$ down to $O\left(10^{-8}\right)$, in particular $D^{0}, D_{(s)}^{+}$and $\Lambda_{c}^{+}$ decay modes. However, the Belle collaboration already determined the bound of the branching ratio for $\mathcal{B}\left(D^{0} \rightarrow\right.$ invisibles $) \leq 9.4 \times 10^{-5}$. The authors of Refs. [7] considered in detail general framework of New Physics (NP) in $c \rightarrow u$ invisibles, relying on $S U(2)_{L}$ invariance and data on charged lepton processes [19]. They found that these assumptions allow upper limits as large as few $10^{-5}$, while in the limit of lepton universality, branching ratios can be as large as $10^{-6}$. To consider invisible fermions, having quantum numbers of right-handed neutrinos, one has to use the effective 
Lagrangian

$$
\begin{aligned}
\mathcal{L}_{\mathrm{eff}}= & \sqrt{2} G_{F}\left[c^{L L}\left(\bar{u}_{L} \gamma_{\mu} c_{L}\right)\left(\bar{v}_{L} \gamma^{\mu} v_{L}^{\prime}\right)\right. \\
& +c^{R R}\left(\bar{u}_{R} \gamma_{\mu} c_{R}\right)\left(\bar{v}_{R} \gamma^{\mu} v_{R}^{\prime}\right)+c^{L R}\left(\bar{u}_{L} \gamma_{\mu} c_{L}\right)\left(\bar{v}_{R} \gamma^{\mu} v_{R}^{\prime}\right) \\
& +c^{R L}\left(\bar{u}_{R} \gamma_{\mu} c_{R}\right)\left(\bar{v}_{L} \gamma^{\mu} v_{L}^{\prime}\right)+g^{L L}\left(\bar{u}_{L} c_{R}\right)\left(\bar{v}_{L} v_{R}^{\prime}\right) \\
& +g^{R R}\left(\bar{u}_{R} c_{L}\right)\left(\bar{v}_{R} v_{L}^{\prime}\right)+g^{L R}\left(\bar{u}_{L} c_{R}\right)\left(\bar{v}_{R} v_{L}^{\prime}\right) \\
& +g^{R L}\left(\bar{u}_{R} c_{L}\right)\left(\bar{v}_{L} v_{R}^{\prime}\right)+h^{L L}\left(\bar{u}_{L} \sigma^{\mu \nu} c_{R}\right)\left(\bar{v}_{L} \sigma_{\mu \nu} v_{R}^{\prime}\right) \\
& \left.+h^{R R}\left(\bar{u}_{R} \sigma^{\mu v} c_{L}\right)\left(\bar{v}_{R} \sigma_{\mu \nu} v_{L}^{\prime}\right)\right]+ \text { h. c.. }
\end{aligned}
$$

In Ref. [7] right-handed massless neutrinos are considered. We analysed massive right-handed fermions and used the notation $v_{R} \equiv \chi_{R}$. One of coloured scalars (see Table I of [18]) which can generate the transition $c \rightarrow u \chi \bar{\chi}$ at the tree level, have following Lagrangian,

$$
\mathcal{L}\left(\bar{S}_{1}\right) \supset \bar{y}_{1 i j}^{R R} \bar{u}_{R}^{C i} \chi_{R}^{j} \bar{S}_{1}+\text { h.c.. }
$$

generates the effective Lagrangian

$$
\mathcal{L}_{\text {eff }}=\sqrt{2} G_{F} c^{R R}\left(\bar{u}_{R} \gamma_{\mu} c_{R}\right)\left(\bar{\chi}_{R} \gamma^{\mu} \chi_{R}\right)
$$

with

$$
c^{R R}=\frac{v^{2}}{2 M_{\bar{S}_{1}}^{2}} \bar{y}_{1 c \chi}^{R R} \bar{y}_{1 u \chi}^{R R *} .
$$

In Tables 1, 2, and 3 we show the bounds on the $\mathcal{B}\left(D^{0} \rightarrow \chi \bar{\chi}\right), \mathcal{B}\left(D^{0} \rightarrow \chi \bar{\chi} \gamma\right)$, and $\mathcal{B}(D \rightarrow \pi \chi \bar{\chi})$ for three selected values of $m_{\chi}$. In our study [18], we found out that the most robust constraints on $c^{R R}$ are obtained from the $D^{0}-\bar{D}^{0}$ oscillations.

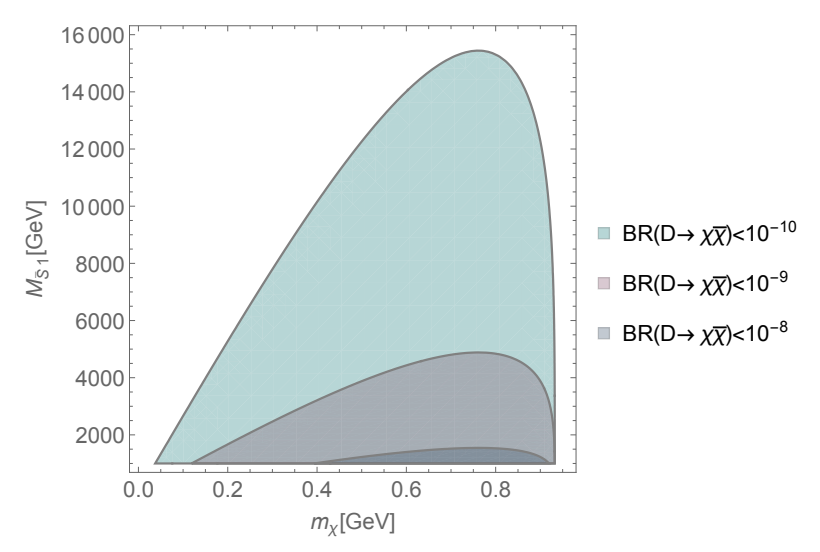

Figure 3: The allowed mass region for $\bar{S}_{1}$ in the range $0<m_{\chi}<\left(m_{D}-m_{\pi}\right) / 2$. The regions are obtained assuming $\mathcal{B}\left(D^{0} \rightarrow \chi \bar{\chi}\right)<10^{-10}, 10^{-9}$ and $10^{-8}$, for the product $\left|\bar{y}_{1 c \chi}^{R R} \bar{y}_{1 u \chi}^{R R *}\right|=1$.

In Fig. 3 we present allowed mass region and find that mass of $\bar{S}_{1}$ can be reachable by LHC. In Table 1 we show the upper bounds on the branching ratio $\mathcal{B}\left(D^{0} \rightarrow \chi \bar{\chi}\right)$ for three selected values 


\begin{tabular}{|c|c|}
\hline$m_{\chi}(\mathrm{GeV})$ & $\mathcal{B}\left(D^{0} \rightarrow \chi \bar{\chi}\right)_{D-\bar{D}}$ \\
\hline 0.2 & $<2.8 \times 10^{-9}$ \\
0.5 & $<1.5 \times 10^{-8}$ \\
0.8 & $<2.3 \times 10^{-8}$ \\
\hline
\end{tabular}

Table 1: Branching ratios for $\mathcal{B}\left(D^{0} \rightarrow \chi \bar{\chi}\right)$ for three selected values of $m_{\chi}$. The constraints from the $D^{0}-\bar{D}^{0}$ mixing is used, with $c^{R R} \leq 5.18 \times 10^{-4}$, assuming $M_{\bar{S}_{1}}=1000 \mathrm{GeV}$.

\begin{tabular}{|c|c|c|}
\hline$m_{\chi}(\mathrm{GeV})$ & $\mathcal{B}\left(D^{0} \rightarrow \chi \bar{\chi} \gamma\right)_{D-\bar{D}}$ & $\mathcal{B}\left(D^{0} \rightarrow \chi \bar{\chi} \gamma\right)_{\text {Belle }}$ \\
\hline 0 & $<3.9 \times 10^{-12}$ & - \\
0.2 & $<3.0 \times 10^{-12}$ & $<1.3 \times 10^{-7}$ \\
0.5 & $<1.0 \times 10^{-12}$ & $<6.3 \times 10^{-9}$ \\
0.8 & $<5.4 \times 10^{-14}$ & $<2.2 \times 10^{-10}$ \\
\hline
\end{tabular}

Table 2: Bounds on the branching ratio for $\mathcal{B}\left(D^{0} \rightarrow \chi \bar{\chi} \gamma\right)$. In the second column we use, the constraint from the $D^{0}-\bar{D}^{0}$ mixing assuming $M_{\bar{S}_{1}}=1000 \mathrm{GeV}$. We use the Belle bound $\mathcal{B}\left(D^{0} \rightarrow\right.$ missing energy $<9.4 \times 10^{-5}$ in the third column. With this bound the Wilson coefficient for $m_{\chi}=0$ cannot be fixed, reflecting in the third column's missing bound.

\begin{tabular}{|c|c|c|}
\hline$m_{\chi}(\mathrm{GeV})$ & $\mathcal{B}\left(D^{0} \rightarrow \pi^{0} \chi \bar{\chi}\right)_{D-\bar{D}}$ & $\mathcal{B}\left(D^{+} \rightarrow \pi^{+} \chi \bar{\chi}\right)_{D-\bar{D}}$ \\
\hline 0 & $<1.5 \times 10^{-8}$ & $<5.8 \times 10^{-8}$ \\
0.2 & $<1.2 \times 10^{-8}$ & $<6.1 \times 10^{-8}$ \\
0.5 & $<6.6 \times 10^{-9}$ & $<3.3 \times 10^{-8}$ \\
0.8 & $<3.0 \times 10^{-10}$ & $<1.5 \times 10^{-9}$ \\
\hline
\end{tabular}

Table 3: Branching ratios for $\mathcal{B}(D \rightarrow \pi \chi \bar{\chi})$. In the second and the third columns the constraint from the $D^{0}-\bar{D}^{0}$ mixing is used, assuming the mass of $M_{\bar{S}_{1}}=1000 \mathrm{GeV}$. In the case $m_{\chi}=0.2 \mathrm{GeV}$, the cut in integration variable is done by taking $q_{c u t}^{2}$, as described in the paper [20]

of $m_{\chi}=0.2,0.5,0.8 \mathrm{GeV}$, using the constraints from the $D^{0}-\bar{D}^{0}$ mixing, $c^{R R} \leq 5.18 \times 10^{-4}$, with $M_{\bar{S}_{1}}=1000 \mathrm{GeV}$. We have calculated branching ratios for $D^{0} \rightarrow \chi \bar{\chi}, D^{0} \rightarrow \chi \bar{\chi} \gamma$ and $D \rightarrow \pi \chi \bar{\chi}$. We point out that the charm meson mixing severely constrain the branching ratio $D^{0} \rightarrow \chi \bar{\chi}$. The bound derived from experimental bound on the branching ratio $D^{0} \rightarrow$ missing energy is up to three orders of magnitude weaker than one coming from the charm mixing. The branching ratios for $D \rightarrow \pi \chi \bar{\chi}$, based on charm mixing constraint, are of the order $10^{-8}$, and therefore hopefully suitable for searches at future tau-charm factories. Current experiments BESIII and Belle II experiments can reach a sensitivity of the order $10^{-6}$.

\section{Summary and outlook}

In the last few years, several necessary steps were done in search of NP in charm semileptonic decays. The SM contributions were carefully studied and are now well known for $c \rightarrow u \ell^{+} \ell^{-}$ decays. The long-distance contributions are calculated, although using the existing vector meson resonance approach. Hopefully, the lattice community will provide us with precise knowledge of hadronic quantities. Lepton flavour universality in rare charm semileptonic decays was questioned 
too, but with the existing experimental data, no significant deviations from the SM was found [8]. In the last two years, charm meson decays to invisibles attracted several studies. Although experimental bounds are not very restrictive, search for such decay rates offers unique null tests of the SM.

\section{Acknowledgment}

The work of SF was in part financially supported by the Slovenian Research Agency (research core funding No. P1-0035).

\section{References}

[1] G. Burdman, E. Golowich, J. L. Hewett and S. Pakvasa, Phys. Rev. D 66, 014009 (2002) doi:10.1103/PhysRevD.66.014009 [arXiv:hep-ph/0112235 [hep-ph]].

[2] S. Fajfer, S. Prelovsek and P. Singer, Phys. Rev. D 64, 114009 (2001) doi:10.1103/PhysRevD.64.114009 [arXiv:hep-ph/0106333 [hep-ph]].

[3] A. Paul, I. I. Bigi and S. Recksiegel, Phys. Rev. D 83, 114006 (2011) doi:10.1103/PhysRevD.83.114006 [arXiv:1101.6053 [hep-ph]].

[4] G. Burdman, E. Golowich, J. L. Hewett and S. Pakvasa, Phys. Rev. D 52, 6383-6399 (1995) doi:10.1103/PhysRevD.52.6383 [arXiv:hep-ph/9502329 [hep-ph]].

[5] S. Fajfer and N. Košnik, Eur. Phys. J. C 75, no.12, 567 (2015) doi:10.1140/epjc/s10052-0153801-2 [arXiv:1510.00965 [hep-ph]].

[6] M. Golz, G. Hiller and T. Magorsch, [arXiv:2107.13010 [hep-ph]].

[7] R. Bause, H. Gisbert, M. Golz and G. Hiller, Phys. Rev. D 103, no.1, 015033 (2021) doi:10.1103/PhysRevD.103.015033 [arXiv:2010.02225 [hep-ph]].

[8] R. Bause, M. Golz, G. Hiller and A. Tayduganov, Eur. Phys. J. C 80, no.1, 65 (2020) [erratum: Eur. Phys. J. C 81, no.3, 219 (2021)] doi:10.1140/epjc/s 10052-020-7621-7 [arXiv:1909.11108 [hep-ph]].

[9] S. De Boer and G. Hiller, Phys. Rev. D 98, no.3, 035041 (2018) doi:10.1103/PhysRevD.98.035041 [arXiv:1805.08516 [hep-ph]].

[10] A. Angelescu, D. Bečirević, D. A. Faroughy, F. Jaffredo and O. Sumensari, [arXiv:2103.12504 [hep-ph]].

[11] S. Fajfer, PoS ICHEP2018, 715 (2019) doi:10.22323/1.340.0715

[12] S. de Boer, Eur. Phys. J. C 77, no.11, 801 (2017) doi:10.1140/epjc/s10052-017-5364-x [arXiv:1707.00988 [hep-ph]]. 
[13] R. Aaij et al. [LHCb], Phys. Lett. B 725, 15-24 (2013) doi:10.1016/j.physletb.2013.06.037 [arXiv:1305.5059 [hep-ex]].

[14] R. Aaij et al. [LHCb], Phys. Lett. B 724, 203-212 (2013) doi:10.1016/j.physletb.2013.06.010 [arXiv:1304.6365 [hep-ex]].

[15] R. Aaij et al. [LHCb], Phys. Rev. Lett. 119, no.18, 181805 (2017) doi:10.1103/PhysRevLett.119.181805 [arXiv:1707.08377 [hep-ex]].

[16] R. Aaij et al. [LHCb], Phys. Rev. Lett. 121, no.9, 091801 (2018) doi:10.1103/PhysRevLett.121.091801 [arXiv:1806.10793 [hep-ex]].

[17] A. Badin and A. A. Petrov, Phys. Rev. D 82, 034005 (2010) doi:10.1103/PhysRevD.82.034005 [arXiv: 1005.1277 [hep-ph]].

[18] S. Fajfer and A. Novosel, Phys. Rev. D 104, no.1, 015014 (2021) doi:10.1103/PhysRevD.104.015014 [arXiv:2101.10712 [hep-ph]].

[19] R. Bause, H. Gisbert, M. Golz and G. Hiller, Phys. Rev. D 101, no.11, 115006 (2020) doi:10.1103/PhysRevD.101.115006 [arXiv:2004.01206 [hep-ph]].

[20] S. Fajfer and A. Novosel, Phys. Rev. D 104, no.1, 015014 (2021) doi:10.1103/PhysRevD.104.015014 [arXiv:2101.10712 [hep-ph]].

[21] S. Fajfer, N. Kosnik and S. Prelovsek, Phys. Rev. D 76, 074010 (2007) doi:10.1103/PhysRevD.76.074010 [arXiv:0706.1133 [hep-ph]].

[22] C. Greub, T. Hurth, M. Misiak and D. Wyler, Phys. Lett. B 382, 415-420 (1996) doi:10.1016/0370-2693(96)00694-6 [arXiv:hep-ph/9603417 [hep-ph]].

[23] S. Fajfer, S. Prelovsek and P. Singer, Eur. Phys. J. C 6, 471-476 (1999) doi:10.1007/s100520050356 [arXiv:hep-ph/9801279 [hep-ph]]. 